\title{
MORPHOLOGICAL INTERPOLATION FOR TEXTURE CODING
}

\author{
Josep R. Casas, Philippe Salembier and Luis Torres \\ Dept. de Teoria del Senyal i Comunicacions \\ Universitat Politècnica de Catalunya \\ Campus Nord UPC - Edifici D5 \\ c/. Gran Capità s/n. \\ 08034 Barcelona, Spain \\ E-mail: josep@tsc.upc.es
}

\begin{abstract}
In this paper a new morphological interpolation technique is presented. It is applied to the coding of the smooth (primary) component in a sketch-based image compression approach for very low bit-rates.

The interpolation technique is intended to perform two dimensional interpolation from any set of initial pixels and, in particular, from sketch data. It makes intensive use of geodesic dilation, a morphological operator that may be implemented by means of FIFO queues. This results in a very efficient process compared to those that perform interpolation by linear filtering on the initial image.

For the application of this method to interpolative image coding, the sketch data is extracted as a set of maximum curvature lines by means of the watershed algorithm. From such information, the interpolation technique obtains a fair reconstruction of both the smooth texture component and the main transitions of the image signal at low bit-rate cost.
\end{abstract}

\section{INTRODUCTION}

Interpolative coding techniques are based on the coding and transmission of a subset of pixels of the original image so that, on the receiver side, the remaining pixels have to be interpolated from the transmitted information alone. The reconstructed image is usually approximated by continuous functions with some permissible error at the interpolated pixels. The positions of the transmitted pixels, called the initial set in the following, may be either a regular subsampling grid or any arbitrary set of points. In the latter case, both the amplitude values and the position of the pixels of the initial set should be coded.

Examples of the application of interpolative coding techniques for arbitrary sets of pixels can be found in [1] and $[2,3]$, where the model of the image used for coding purposes is based on the concept of the "raw primal sketch" [4]. In such model, the image is assumed to be made mainly of areas of constant or smoothly changing intensity separated by discontinuities or edges. Therefore, it is possible to obtain a reconstruction of the original image by coding only

This work has been partly supported by the Spanish Government under contract TIC 92-1319-C03-01-PB and partly by RACE MORPHECO Project 2053 of the European Union the sketch data, which consists of two types of information: the geometric structure of the discontinuities and the amplitudes at the edge pixels. The amplitudes of the pixels in between are then approximated by interpolating their values from the coded information under certain smoothness constraints. The extraction of sketch data from the original image is the key issue in this coding approach. For a given interpolation strategy, the selection and coding of the initial pixels sets the trade-off between the coding cost and the quality of the reconstructed image.

A main drawback that existing interpolation algorithms for the reconstruction of the image from sketch data present is the filtering process they use, with rather high computational load. In this paper, a morphological tool intended to perform two dimensional interpolation from any set of initial pixels is presented. The algorithm is based on the geodesic dilation operator, resulting in a highly efficient process compared to those that perform linear filtering on the initial image. Using this interpolation strategy, pixels with large curvatures (absolute values of the second derivative) are taken as the initial set, and yield an optimal reconstruction of the original image in the minimum distortion sense. Moreover, if the initial pixels are grouped so that the coding of their positions and amplitudes can be made efficiently, this approach is able to reach high compression rates. Some first promising results of the interpolative technique using open contour lines were presented in [5]. In the present paper, it will be shown that this technique have proved to be very efficient for the coding of the smooth texture component in high compression applications using networks of closed lines for the initial set.

\section{MORPHOLOGICAL INTERPOLATION}

The target of the morphological interpolation algorithm is to approximate the amplitudes of unknown pixels of the image by fitting a surface on a subset of pixels of known values (the initial pixels). Such surface is constrained to be maximally smooth between the known pixels in the sense that pixel to pixel variations in the interpolated area should be minimized.

A suitable strategy for $2 \mathrm{D}$ interpolation is to compute at each point the average of the amplitudes of the initial pixels weighted by the inverse of the distances to each of 


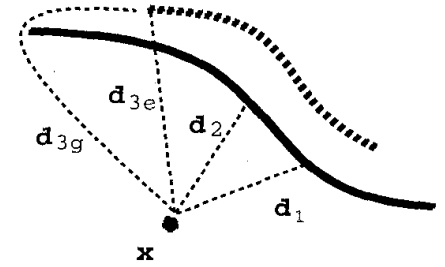

Figure 1: Geodesic distance measure to interpolate pixel $x$

them [6]. The nearest pixels have stronger influence than the distant ones, and the interpolated amplitudes change slowly in the areas in between.

\subsection{Geodesic distance weighting}

The distance measure is taken as the geodesic distance within the set of unknown pixels, that is, the length of the shortest path joining two points which is completely included within the set of unknown pixels. The use of the geodesic distance allows the preservation of the transitions imposed by the initial set. This is illustrated in Fig. 1. The set of initial pixels is indicated by thick solid and dashed lines. Let us suppose that the dashed line represents the upper edge of a spatial transition and the solid line represents the lower edge. The influence of the amplitude values of the upper edge (dashed line) at pixel $x$ is given by the inverse of the geodesic distance $d_{3 g}$, which is larger than the Euclidean distance $d_{3 e}$. Therefore, the interpolated values at pixel $x$ will be mainly influenced by the initial pixels of the solid line because the weights of the pixels located on the other side of the transition, at a larger geodesic distance, will be much smaller. As a result, the use of the geodesic distance allows the preservation of the transitions.

The interpolation technique may be efficiently implemented by an iterative algorithm based on morphological operators. Each iteration of the algorithm performs two basic steps, namely a geodesic propagation process followed by a smoothing step.

\subsection{Geodesic propagation process}

For the geodesic propagation process, instead of computing geodesic distances from all the unknown pixels to every point of the initial set, the amplitude values of the known pixels are propagated by geodesic dilation to fill the empty areas of the image. This is performed by a fast algorithm, using FIFO queues, so that each pixel is treated only once in a complete propagation process. Fig. 2 shows some intermediate images corresponding to the propagation process from the initial set. It can be observed that, at the positions where two or more propagation fronts originated from initial pixels of different amplitudes meet, the process stops and a false transition is created. These false transitions appearing outside the set of initial pixels will be smoothed by the smoothing step.

\subsection{Smoothing step}

The morphological Laplacian ${ }^{1}$ is used as a transition detector in order to obtain the false transitions. Pixels on both sides of the false transitions compose the set of secondary pixels. A grey level value equal to the average of the grey level values on both sides of the transition is assigned to each secondary pixel. This is the smoothing step. Secondary pixels will be used in the next iteration of the algorithm in order to smooth out these transitions.

Then, a second iteration is performed: the propagation step propagates the grey level values from the sets of initial as well as secondary pixels. The propagation creates new false transitions which define a new set of secondary pixels where grey level values are smoothed. Note that this new set of secondary pixels generally does not include the first set of secondary pixels. This process of 1) propagation of values from the initial and secondary pixels, and 2) smoothing of the grey levels of the new secondary pixels, is iterated until idempotence.

Fig. 3 shows the images resulting from the geodesic propagation process for some iterations of the interpolation algorithm. Observe the progressive smoothing of the false transitions. After a few number of iterations, the algorithm quickly converges to the final interpolated image.

\section{IMAGE CODING BY MAXIMUM AND MINIMUM CURVATURE LINES}

The application of the morphological interpolation algorithm to image coding relies on the selection of a proper set of initial pixels. Given that the interpolative method only generates smooth surfaces between the initial pixels, such pixels should be selected so that the main transitions between these surfaces are kept. The initial pixels should, at the same time, allow a good restoration of the image by interpolation and lead to a compact representation. In this section an efficient image representation based on networks of lines is presented. Networks of lines are interesting because they can be efficiently coded using derivative chain code techniques.

\subsection{Extraction of the sketch data}

The main difficulty consists in selecting lines that can lead to a good restoration of the image. A first idea may be to select the 'crest' and 'valley' lines of the original image, that is the watershed [7] of the image and of its dual. However, some experiments have shown that, using such lines as initial set, the resulting image obtained by the morphological interpolation technique lacks of transitions. This is because the information obtained from the image extrema does not allow to locate the important transitions precisely. The set

\footnotetext{
${ }^{1}$ The morphological Laplacian is defined as the residue of the gradients by dilation $(\delta)$ and erosion $(\varepsilon)$. Gradient by dilation: $g^{+}(f)=\delta(f)-f$. Gradient by erosion: $g^{-}(f)=f-\varepsilon(f)$. Morphological Laplacian: $L(f)=g^{+}(f)-g^{-}(f) . L(f)$ is greater than zero at the lower edge of the transitions and smaller than zero at the upper edge. In flat surfaces or slanted planes without convexity changes, it cancels out. Indeed, it can be shown that the morphological Laplacian is an approximation of the signal second derivative.
} 
of points with the largest curvature values characterizes the upper and lower edges of sharp transitions and locate their position more precisely. Thus, these points are proposed as the initial set for the interpolation.

In order to extract lines of maximum and minimum curvature the following steps are carried out. First, the original image is prefiltered by a dynamics prefilter [8], which removes low contrasted components while preserving the grey level shape of the remaining components. It serves for removing most of the noisy and low amplitude edges of the original image. Second, the morphological Laplacian is computed. Indeed, it can be shown that the morphological Laplacian is an approximation of the signal second derivative, and its crest and valley lines correspond to the lines of largest curvature of the image. Third, the watershed applied on the Laplacian extracts a network of lines identifying the lines of maximum curvature. The same process, on the dual of the Laplacian, extracts the lines of minimum curvature. In order not to have too many irrelevant curvature lines, a set of markers is needed to compute the watershed of the Laplacian. These markers indicate flat and smooth areas of the original image, where it is not necessary to extract any transition line.

The extraction process is illustrated in Fig. 4. The upper row shows the original image, the prefiltered image and the Laplacian of the latter. In the left image of the lower row, the set of markers are presented in dark grey, and the result of the two watershed processes (the networks of maximum and minimum curvature lines) are drawn in white and black respectively.

\subsection{Coding of the sketch data}

The information about the position of the networks' pixels and the grey level values of these pixels in the original image have to be coded and transmitted to the receiver. Since the networks are composed of connected pixels, a derivative chain code can be used to code the pixels' positions.

The grey level values are coded by polynomial approximation. More precisely, the network is broken at each triple point (points with more than two branches) and the grey level values of the pixels located under the resulting curves are approximated by a second order polynomial. The three coefficients that define each polynomial are quantized, entropy coded and transmitted. At the receiver, the networks of lines with the approximated grey levels are used as initial pixels for the interpolation. These values and the restored image are presented, respectively, in the last two images of the lower row in Fig. 4.

\section{RESULTS AND CONCLUSION}

The compression ratio achieved with the above strategy is equal to 30 for the interpolation result shown in the last image of Fig. 4. In this example, 178 (175) maximum (minimum) curvature lines have been extracted. The position information has been coded by chain code with a cost of the $55 \%$ of the total coding rate, while the remaining rate $(45 \%)$ corresponds to the quantized coefficients of the polynomial approximation. It should be pointed out that the high degree of correlation that exists between the positions and amplitude levels of the curves that carry information of the lower and upper edge of the same transition has not been exploited in the coding process, what may permit an extra increase of the compression ratio.

As can be observed, most of the smooth texture component has been nicely reconstructed. Sharp transitions are produced by those curves of the initial set having high level amplitudes which are very close to curves of low level amplitudes, whereas variations of low contrast are restored either by the interpolation algorithm in the pixels in between or by the smooth variations coded in the amplitude profiles on the network lines.

The interpolation technique that has been presented is general in the sense that it is able to perform interpolation from any set of given pixels, for instance, from a set of isolated points or isolated lines of open [5] contours. As the closed networks of the initial image suggest, this coding approach may be used as well for texture coding in a segmentation-based scheme. However, only a primary component consisting of smooth textures and sharp transitions may be restored. For lower compression ratios and higher qualities, some form of waveform coding (VQ, SBC) should be used to encode the fine textures of the coding residue.

\section{REFERENCES}

[1] S. Carlsson. Sketch based coding of grey level images. EURASIP, Signal Processing, 15(1):57-83, July 1988.

[2] X. Ran and N. Farvardin. A perceptually motivated three-component image model. Part I: Description of the model. IEEE Transactions on Image Processing, 4(4):401-415, April 1995.

[3] X. Ran and N. Farvardin. A perceptually motivated three-component image model. Part II: Application to image compression. IEEE Transactions on Image Processing, 4(4):430-447, April 1995.

[4] D. Marr. Vision. Freeman, New York, 1982.

[5] J.R. Casas and L. Torres. Feature-based video coding using mathematical morphology. In EURASIP, editor, EUSIPCO 94, VII European Signal Processing Conference, pages 143-146, Edinburgh, U.K., September 1994.

[6] P. Soille. Spatial distributions from contour lines: an efficient methodology based on distance transformations. Journal of Visual Communication and Image Representation, 2(2):138-150, June 1991.

[7] F. Meyer and S. Beucher. Morphological segmentation. Journal of Visual Communication and Image Representation, 1(4):21-46, September 1990.

[8] M. Grimaud. A new measure of contrast: the dynamics. In SPIE, editor, Visual Communications and Image Processing'92, volume 1769, pages 292-305, San Diego, CA, July 1992. 


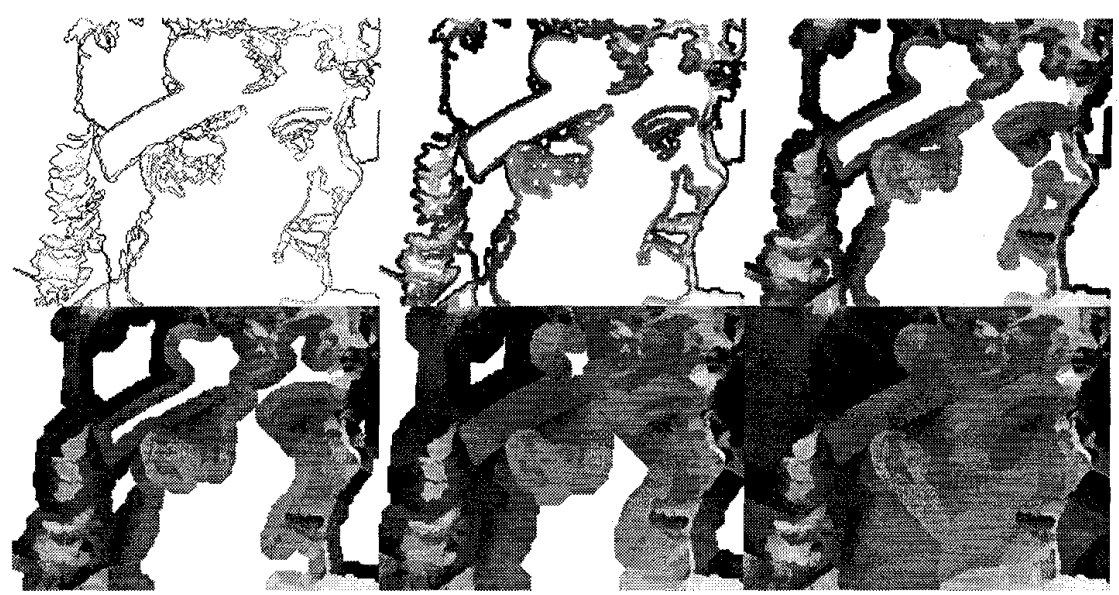

Figure 2: Geodesic propagation process from an initial set of pixels

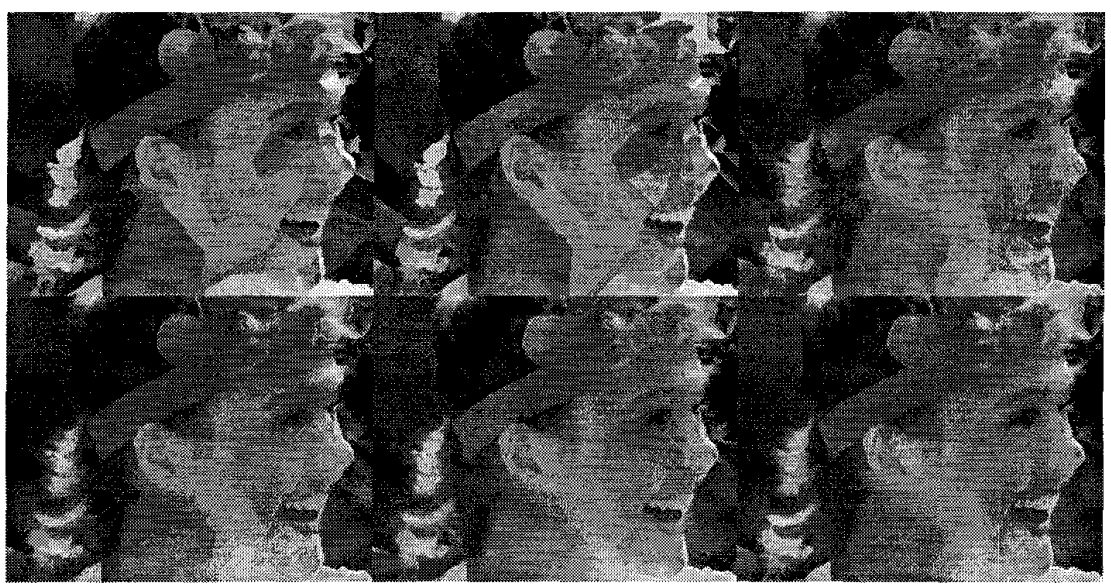

Figure 3: Iterations (1st, 2nd, 4th, 6th, 8 th and 9 th) of the morphological interpolation process

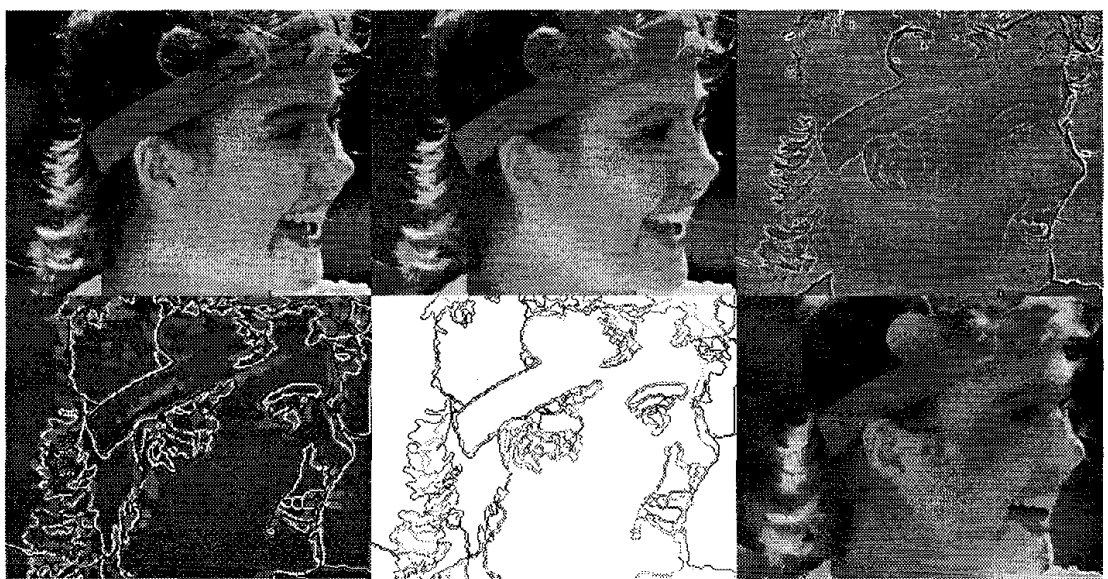

Figure 4: Morphological interpolation for coding. Upper row: original, prefiltered and Laplacian. Lower row: markers and watershed lines, initial pixels and interpolated image (compression 30 ). 